\title{
Cognitive and Pragmatic Motivations for the Dissymmetry between Deictic Expression and Denoting Expression in Person Deixis
}

\author{
Hongwei Zhao \\ School of Humanity and law, North China University of Technology, Beijing, China \\ Email: jameszhao456@163.com
}

\begin{abstract}
This paper aims at explaining the dissymmetry between deictic expression and denoting expression under the frame of Markedness Theory, in terms of which the opposition between marked term and unmarked term is motivated by cognitive and pragmatic factors. The paper first distinguishes deictic expression from denoting expression, and then defines the dissymmetry between deictic expression and denoting expression in the category of person deixis. Finally, the paper explores the pragmatic and cognitive motivations for the dissymmetry under the frame of Markedness Theory. Examples in this paper reveal that, in the course of people's cognition, participants take themselves as reference points to achieve referring, and the dissymmetry of the use of person deixis is determined by its accessibility in speakers' mind; from the pragmatic angle, observing pragmatic principles is another reason for the dissymmetry between deictic expression and denoting expression in person deixis.
\end{abstract}

Index Terms - dissymmetry, person deixis, accessibility, pragmatic principles

\section{DEIXIS}

Deixis has long been the central concern in pragmatics, referring to the fact that interpretation of certain linguistic expressions in an utterance depends on contextual information. The context concerned is the knowledge relative to the immediate or actual speech situation utterances occur in, such as the time and location of the conversation, topic, participants, non-verbal actions they are performing, and immediately observed objects or events. Specifically, deixis serves to indicate a certain relation between linguistic expressions and speech context, identifying the speaker and hearer, the location and the time of the utterance.

Lyons (1968, 1977), Fillmore (1971, 1975), Levinson (1983), He Zi-ran (988, 1997), Zhang Quan (1994), He Zhao-xiong (2000), Jiang Wang-qi (2000), and other scholars have made a detailed study on deixis.

Lyons (1977) gives a definition to Deixis in his Semantics referring to "the function of personal and demonstrative pronouns, of tense and of a variety of other grammatical and lexical features which relate utterances to the spatio-temporal co-ordinates of the act of utterance." (p.636). He discusses person, space and time deixis and proposes the phenomenon of deictic projection. But his study lacks further exploration.

Fillmore (1971) regards deixis as some lexical and grammatical units that can be understood only when they are related to social context to identify the speech participants and the spatio-temporal orientation of the speech event. He makes a study of time, space, discourse and social deixis and holds that there are deictic and non-deictic usages for deixis.

Levison has a detailed study of deixis from philosophical and descriptive aspects in his Pragmatics published in 1983. He also points out the phenomenon of pre-emptiveness of deixis. He holds that pre-emptive nature of deixis refers to the phenomenon that deixis is used prior to its corresponding non-deixis, but he doesn't explore this phenomenon further.

Chinese scholar Zhang Quan (1994) pays attention to the phenomenon of pre-emptiveness, makes a deep research on it, and brings up the anti-pre-emptiveness that non-deictic expression is used prior to deictic expression in some situations. However, his study is limited to the pre-emptiveness and anti-pre-emptiveness in the category of time deixis, and there is no any systemic interpretation to the phenomenon.

He Zhaoxiong (2000) gives a definition of deixis and distinguishes deictic usage and non-deictic usage. He thinks that the point of reference is a necessary item for understanding the usage of deixis. He puts forward deictic usage and no-deictic usage of deixis, but there is no further exploration to them.

Pre-emptiveness of deixis in speech act brings about the dissymmetry between deictic expression and denoting expression (non-deictic expression). With the framework of Markedness Theory, it seems possible to make a systematic explanation to the dissymmetry between deictic expression and denoting expression in the categories of deixis. From pragmatic and cognitive aspects, Markedness Theory aims to explain how the dissymmetry comes into being.

\section{Dissymmetry In CATEGORIES OF DeIXIS}


Dissymmetry can be found in all aspects of language, such as phonetics, word-formation, syntax, pragmatics and so on. It refers to the opposition between marked term and unmarked term within certain category. In the categories of deixis, deictic expressions and denoting expressions referring to or denoting the same object are used dissymmetrically, which is called the phenomenon of dissymmetry. Specifically, when the speaker in speech acts denotes or refers to the person, object, time and place in the domain of reference, deictic expression is used more frequently than denoting expression which forms the dissymmetry. On the one hand the dissymmetry between the use of deictic expressions and denoting expressions is the psychological tendency of speakers in the process of selecting deixis, on the other hand it results from the speaker's observing pragmatic principles in speech acts.

It is necessary to distinguish denoting from referring in the categories of deixis. John Lyons (1977) separates the terms refer and denote. Denote is used for the relationship between a linguistic expression and the world, while refer is used for the action of a speaker in picking out entities in the world.

We connect language forms with the corresponding entities in the world by denoting. The abstract symbols in languages gain their practical meanings in verbal communication by denoting the entities in context. Denoting expressions are composed of the names of objects, including proper nouns (New York, Einstein), definite descriptions (the man with a book, the scientist) and common nouns. While referring sets up connection between deictic expression and the context with the speaker as reference point, which forms a domain of reference around the speaker. All the persons, objects, places, time within the domain of reference get confirmed by deictic expressions with reference to the speaker.

\section{DeICTIC EXPRESSIONS AND DENOTING EXPRESSIONS IN THE CATEGORY OF PERSON DeIXIS}

\section{A. The Features of Person Deixis}

In speech acts, the speaker occupies the reference center and set up a frame of reference (a domain of reference) around herself, within which entities are identified by deictic expressions with reference to the speaker. However, as for the entities beyond the domain of reference, denoting expressions are used to achieve denotation. On the one hand, the feature of Self-sufficiency decides the use of deictic expression is the first choice. On the other hand, the use of denoting expressions is decided by the feature of openness of person deixis.

Self-sufficiency means that speech act is a self-contained system which is composed of the speaker, the hearer and overhearers who hear the utterance but not directly addressed. In the self-contained system, the speaker is the reference center and casts herself in the role of ego and takes everything from her viewpoint. In this case, person deictic expression is used for referring to the referent, but in a particular context, non-deictic expression functioning as denoting is used.

Openness refers to the fact that the uncertainty of the referents or entities within a closed self-contained system makes non-deictic expression use possible. Based on the context dependency of person deixis, the referents constantly change with different contexts, which makes the self-contained system an open one.

\section{B. Deictic Expression and Denoting Expression}

Deictic expression and denoting expression are used to set up connection between language symbols and entities by referring and denoting within the categories of deixis.

1. Deictic Expression

Deictic expression is the language form which carries certain context features and has to be interpreted in the context, whose referent is decided by context. The information about person, time, place carried by deictic expression has to be inferred in connection with contextual information. For example, the sentence: "I will wait for you here half an hour later." in which "I" refers to the speaker, "here" to the place where the speaker is speaking, "half an hour later" can be inferred with the time when the utterance is happening as reference point. In this case, one can not interpret the meaning of the utterance without turning to a context. The interpretation of deictic expression in an utterance depends on physical environment which is concerned with the occasion where an event happens. Physical environment is related to short-term memory of human beings and is easily extracted. For example,

(1) Look! She is walking over to us.

With the help of physical environment, "She" sets up its relation with the referent which is also maximally accessible because it is physically present in the context of the utterance and stored in the short-memory of human beings.

2. Denoting Expression

Denoting expression includes proper nouns (New York, Einstein), definite descriptions (the man with a book, the scientist) and common nouns (desk, cup, TV). Denoting expression can be interpreted with the help of encyclopedic knowledge, which is stored in long-term memory of human beings as a kind of knowledge schema. Once people meet proper nouns or definite descriptions or common nouns, they will search the encyclopedic knowledge in their brains for the corresponding images with the same features as denoting expressions. For example,

(2) Shakespeare was perhaps the greatest writer in the world.

If the encyclopedic knowledge about Shakespeare is stored in the hearer's brain, he/she can radically identify Shakespeare as a dramatist who ever made great contribution to the world.

(3) The lady who died in a car accident when attempting to escape photographers in a high-speed chase through Paris 
was beloved by millions for her humanitarian concerns.

When the definite description "The lady who died in a car accident when attempting to escape photographers in a high-speed chase through Paris" comes to the hearer, he/she will search the encyclopedic knowledge in their memory system for a lady who died from getting out of photographers' chasing. It is easy for people who have information about the lady to identify her as the princess of Wales. Therefore, denoting expression achieves its denotation by connecting with encyclopedic knowledge environment.

\section{Pragmatic and Cognitive Approaches to the Dissymmetry in the Category of Person Deixis}

The Markedness Theory is put forward by the Prague School in the 1930s, and regarded as a significant basic principle in analyzing language system in linguistics. Markedness Theory has a strong explanatory power to various dissymmetries in language which holds the dissymmetry of internal members in a category is the opposition of the marked and the unmarked term. The unmarked term is described as more "natural" phenomenon, which carries neutral meaning, while the marked term is described as a less "natural" phenomenon which carries some special meaning. The unmarked term spreads more broadly than the marked term, and the frequency of the use of the unmarked term is beyond that of the marked term.

As is mentioned, the interpretation of deictic expression relies on physical environment, and its referent is stored in the short-term memory of people and can be extracted very fast. However, the interpretation of denoting expression relies on encyclopedic knowledge, and the corresponding image is stored in the long-term memory of people and extracted with more time and energy. In a domain of reference, deictic expression which is used more frequently and spreads more broadly is the unmarked term; denoting expression is the marked term.

Markedness Theory attributes the dissymmetry in language to pragmatic and cognitive factors. The dissymmetry in the process of using deictic expression and denoting expression can also be explained from the perspectives of pragmatics and cognition.

\section{A. Accessibility and Dissymmetry}

One feature of deixis is taking the speaker as a reference center and the use of deixis is determined by the degree of it's accessibility in speakers' mind. Accessibility is a psychological concept and stands for the degree of convenience to which language users extract schema knowledge from the memory system in brains or the speed to activate knowledge in brains. Moreover, the time that different types of deixis take to activate the corresponding knowledge in brains is different.

Craik and Lockhart (1972) hold that memory system of human beings is composed of short-term memory and long-term memory. Ariel (1988) believes that the differences between the two types of memory rely on the different levels of activation in brains.

The accessibility system of deixis is a continuum organized by "the degree of accessibility", which can be divided into three levels: high level, medium level and low level. Ariel (1990) names deixis "high accessibility marker", "medium accessibility marker", and "low accessibility marker" (p. 56). The simpler the structure of deixis is, the less information it carries, the higher degree of accessibility is, vice versa. Low accessibility markers are the deixis which have to be inferred from "encyclopedic environment". As we all know, encyclopedic knowledge contains a large amount of semantic information and is activated with great effort, whose degree of accessibility is relatively low. Low accessibility markers mainly include definite descriptions, proper nouns and common nouns with general concepts. Medium accessibility markers principally include a variety of deictic expressions which can be interpreted under physical environment. High accessibility markers refer to those deixis interpreted against language environment, mainly including zero (Ariel, 1990). Ariel (1994) makes an accessibility marking scale as follows. (deixis on the left side have the highest accessibility)

Zero $>$ unstressed pronouns $>$ stressed pronouns $>$ proximal demonstrative $+\mathrm{NP}>$ distal demonstrative $+\mathrm{NP}>$ first name $>$ last name $>$ short definite description $>$ long definite description $>$ full name $>$ full name + modifier

As is mentioned, deictic expression is interpreted against physical environment, while denoting expression is interpreted against encyclopedia environment. In this case, according to accessibility marking scale of deixis, deictic expressions fall into medium accessibility markers and denoting expressions fall into low accessibility markers. For example,

(4) I went to cinema this afternoon.

James went to cinema this afternoon.

The person standing in front of you went to cinema this afternoon.

Among the three sentences with the same meaning, why do we usually use "I" for self instead of the name "James" or "the person standing in front of you"? Accessibility theory gives a satisfying explanation. When you are speaking with others, you are an entity in physical environment and have extremely high accessibility. Therefore, high accessibility marker (pronoun) is used here for referring. Definite descriptions and full names are low accessibility markers, so "the person standing in front of you" can't be used in the situation. It is clear that the selection of deixis depends on its degree of accessibility in speakers' mind.

\section{B. Pragmatic Principles and Dissymmetry}


Apart from cognitive reason for the dissymmetry, observing pragmatic principles is one of reasons for the dissymmetry, which include economic principle, cooperative principle and politeness principle.

1. Economic Principle and Dissymmetry

Leech (1983) illustrates the economic principle from the perspective of language use, referring to the fact that participants in speech acts are always intending to convey information accurately with minimum efforts. observing economic principle in the process of selecting deixis is one of reasons for the phenomenon of dissymmetry between deictic expression and denoting expression.

With componential analysis, deictic expression and denoting expression in the category of person deixis can be analyzed as followings:

Deictic expression in person deixis: [ego] [referring] [the entities in a domain of reference] [physical distance]

Denoting expression in person deixis: [naming] [denoting] [the entities out of a domain of reference] [the corresponding relation in features between language forms and entities]

As is shown in the former componential analysis, deictic expression sets up far or near relation between the speaker itself and the entities with the speaker as a reference point. Since the referent is the participant in speech acts, to set up the relation between self and the entity only needs to identify the distance between the referent and the speaker against physical environment. The referent which is physically present in the context of the utterance is also maximally accessible and can be identified with less time and energy. The participants near to the speaker are symbolized as "you"; the participants away from the speaker are symbolized as "he (him, she, her, it), they (them)".

In the latter componential analysis, what is different from the componential analysis for person deictic expression is that we need to establish the connection between denoting expression and the entity in real world. With conceptual meaning of denoting expression, hearers identify the entity by recognizing the features of the corresponding image in their mind, which is called semantic recognition. In fact, building up the connection between denoting expressions and target entities is to conceptualize target entities. Since the interpretation of denoting expression is achieved with the help of encyclopedic knowledge which is a type of schema in long-term memory, much time and energy needs putting in to extract the image related to denoting expression. What's more, in view of different encyclopedic environments of hearers, identifying the wrong image in hearers' encyclopedic environments may lead to failure of communication. Therefore, from the perspective of economic principle, we usually use person deictic expressions to refer to the participants in speech acts. For example,

(5) He is my good friend. (He= Bai Yansong)

(6) Bai Yansong is my good friend.

Within the domain of reference, we usually choose sentence (5). The speaker takes self as a reference point, building the connection between self and target entity against the physical environment of speech act, finally achieving its function of referring with less energy.

In the domain of reference, Sentence (6) is considered improper. On the one hand, interpreting the utterance requires the hearer in speech act to extract some information about Bai Yansong from his/her encyclopedic environment and conceptualize the target entity, finally set up the connection in features between the image and the target entity, which consumes much more time and energy; on the other hand, since there are millions of the name of Bai Yansong, misunderstanding might happen, the hearer may extract an image from his/her encyclopedic environment that Bai Yansong is the famous host from a television station instead of identifying the person as the participant in speech act. In this case the hearer not only wastes a lot of time and effort but also fails to interpret the speaker's meaning. In view of economic principle, speakers tend to choose sentence (5) in which the speaker takes self as a reference point.

2. Cooperative Principle and Dissymmetry

Speech act is a cooperative activity between speakers and hearers, and cooperative principle is one of reasons for the phenomenon of dissymmetry. Cooperative principle functions as adapting what the speaker conveys to make sure the success of communication with the presupposition which both the speaker and the hearer are willing to cooperate with each other.

Within a domain of reference, deictic expression is used in order to avoid misunderstanding between the two sides in speech acts, because the hearer also assumes the speaker will take self as a reference point. Called by name, the hearer in a domain of reference may think there is a person with the same name with him/her out of the spot. So observing cooperative principle can help speakers and hearers avoid misunderstanding.

Self-referring by name seems to take self as a stander-by, which is likely to make the hearer confused. The hearer takes the referent as some person out of the domain of reference by mistake and fails to interpret what the speaker tends to express, which finally causes the failure of communication. The utterance can be interpreted correctly on the assumption that both sides in speech acts observe cooperative principle.

The hearer and the speaker in speech act communicate with each other on the assumption of observing cooperative principle. In the process of interpretation, the hearer assumes that the speaker will observe cooperative principle, try to estimate what the hearer knows and package her material accordingly, and certainly observe the maxim of quality, the maxim of quantity, the maxim of manner and the maxim of relevance. In consideration of cooperative principle, the speaker will say something easily understood by the hearer and incline to use person deictic expression within a domain of reference. 
3. Politeness Principle and Dissymmetry

Politeness is one of principles observed by human beings in speech acts and one of reasons for dissymmetry as well.

In certain language, e.g. Chinese, calling the participants in a domain of reference by names is considered impolite and sounds like a command, therefore violates politeness principle in speech acts.

(8) Wang Yue, shen ti hao dian'er le ma? (Wang Yue, feel better?)

(9) Ni shen ti hao dian'er le ma? (Do you feel better?)

We are likely to choose sentence (9) in speech acts. First, all the participants in the domain of reference assume each observes politeness principle. In this case, the use of name for denoting misleads the hearer to believe that the entity is the person out of the domain of reference and search for the person in her encyclopedic knowledge. Second, calling a person by name in certain languages is considered a quite impolite behavior and widens the distance between two sides psychologically. Therefore, speakers' using deictic expression to refer to the entity within a domain of reference is a normal and unmarked.

\section{CONCLUSION}

This paper aims to explain the dissymmetry between deictic expression and denoting expression in person deixis under the framework of Markedness Theory, in terms of which the opposition between marked term and unmarked term is motivated by cognitive and pragmatic factors. Markedness Theory discusses the root of dissymmetry from the perspectives of pragmatics and cognition. From the aspect of cognition, the dissymmetry in the category of person deixis complies with the universal principle of human exploring the world and the selection of person deixis relies on its accessibility in the speaker's mind; from the perspective of pragmatics, dissymmetry takes shape in the process of selecting deixis on the premise of observing pragmatic principles. The current study only focuses on the dissymmetry in the category of person deixis, in fact the dissymmetry also spreads to the other categories of deixis, which needs to be explored further.

\section{REFERENCES}

[1] Ariel, M. (1988). Referring and accessibility. Journal of Linguistics, 24, 65-67.

[2] Ariel, M. (1990). Accessing noun-phrase antecedents. London: Routledge,

[3] Ariel, M. (1994). Interpreting anaphoric expressions: A cognitive versus a pragmatic approach. Journal of Linguistics, 30, 3-42.

[4] Ariel, M. (1998). The linguistic status of the here and now. In Cognition linguistics, 9, 189-238.

[5] Craik, F. I., \& Lockhart, R. S. (1972). Levels of Processing: A framework for memory research. Journal of Verbal Learning and Verbal Behavior, 12, 599-607.

[6] Croft, William. (1990). Typology and Universals. Cambridge: Cambridge University Press.

[7] Chen Zhi-an, Peng Xuan-wei. (1994). Study on Person Deixis. Journal of Foreign Languages, 3, 28-34.

[8] Fillmore, C. J. (1971). Towards a theory of deixis. The PCCLLU Papers (Department of Linguistics, University of Hawaii), 3 , 219-241.

[9] Fillmore. C. J. (1975). Santa Cruz Lectures on Deixis. Mimeo, Indianna University Linguistics Club.

[10] Gao Yan-mei. (2002). Strata of Reference. Journal of Foreign Languages, 139, 51-56.

[11] He Zhao-xiong. (2000). A New Course Survey on Pragmatics. Shanghai: Shanghai Foreign Languages Education Press.

[12] He Zi-ran. (1988). A General Introduction to Pragmatics. Changsha: Hunan Education Press.

[13] He Zi-ran. (1997). Pragmatics \& English Studies. Shanghai: Shanghai Foreign Languages Education Press.

[14] Jiang Wang-qi. (2000). Pragmatics: Theory \& Applications. Beijing: Peking University Press.

[15] Leech, G.. (1983). Principles of Pragmatics. London: Longman.

[16] Levinson, S. C. (1983). Pragmatics. Cambridge: Cambridge University Press. 54-96.

[17] Levinson, S.C. (2001). Pragmatics. Beijing: Foreign Language Teaching and Research Press.

[18] Lyons, J. (1968). An Introduction to Theoretical Linguistics. Cambridge: Cambridge University Press.

[19] Lyons, J. (1975). Deixis as the Sourse of Reference. In E.L. Deenan (Ed.) Formal semantics of natural language. Cambridge: Cambridge University Press.

[20] Lyons, J. (1977). Semantics. Cambridge: Cambridge University Press.

[21] Sun Lei. (2002). History and Current Situation of the Study of Western Deictic Words. Journal of Sichuan University, 123, 70-75.

[22] Wu Yi-an. (2004). Spatial Demonstratives in English and Chinese. Amsterdam: John Benjamins Pub.

[23] Zhang Quan. (1994). On the Pre-emptive Nature of Deixis. Modern Foreign Languages, 2, 6-12.

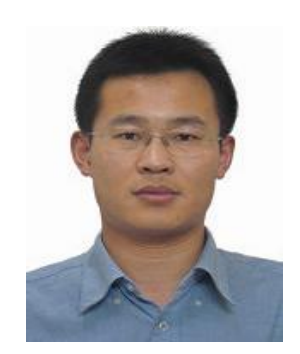

Hongwei Zhao was born in Botou, Hebei Province, China in 1981. He received his M.A. degree in linguistics from Guizhou Normal University, China in 2006.

He is currently a visiting scholar in University of British Columbia and a lecturer in English department, School of Humanity and Law, North China University of Technology, Beijing, China. His research interests include Linguistics \& Applied Linguistics, Pragmatics, Cognitive Linguistics, and etc. 